\title{
A SHARP ESTIMATE FOR DYADIC MARTINGALES WITH MULTIPLE INDICES
}

\author{
GREGORY J. MORROW
}

(Communicated by Bert E. Fristedt)

\begin{abstract}
A variant of Doob's maximal inequality is obtained for dyadic martingales with multiple indices. The inequality furnishes a precise estimate of the $L^{p}$ norm of the maximal function in terms of the $L^{p}$ norms of the jumps, $p \geq 2$.
\end{abstract}

1. Introduction. Let $\left\{f_{n}, \mathcal{F}_{n} ; n \in \mathbf{Z}_{+}^{d}\right\}$ be a real $d$-parameter $L^{2}$ bounded dyadic martingale with difference array $\left\{\Delta_{n} f\right\}$. The underlying probability space is $[0,1)^{d}$ equipped with Lebesgue measure. Here $\mathcal{F}_{n}$ is the field consisting of all rectangles

$$
\prod_{i=1}^{d}\left[\left(k_{i}-1\right) 2^{-n_{i}}, k_{i} 2^{-n_{i}}\right) \subseteq[0,1)^{d}
$$

such that $1 \leq k_{i} \leq n_{i}, i=1, \ldots, d$; with $n=\left(n_{1}, \ldots, n_{d}\right)$. Also, $\Delta_{n} f:=$ $\sum_{n-1 \leq m \leq n} \pm f_{m}$ with $\mathbf{1}=(1, \ldots, 1)$, so that $E\left(\Delta_{n} f \mid \mathcal{F}_{m}\right)=0, m \leq n, m \neq n$. Set $f^{*}:=\sup _{n}\left|f_{n}\right|$. The main result of this note is the following.

THEOREM 1.

$$
\left\|f^{*}\right\|_{p} \leq q^{d}(p-1)^{d / 2} \sqrt{\sum\left\|\Delta_{n} f\right\|_{p}^{2}}, \quad p \geq 2 .
$$

The case $p=2$ is just Doob's $L^{2}$ maximal inequality in the $d$-parameter setting. The main interest for large $p$ lies in the behavior of the best constant in this norm inequality. In fact, for a suitable larger class of $d$-parameter martingales, R. Gundy [4] shows that $\left\|f^{*}\right\|_{p} \leq(C p)^{d}\left\|\left(\sum\left(\Delta_{n} f\right)^{2}\right)^{1 / 2}\right\|_{p}, p \geq 2$, while by Minkowski's inequality the last square function norm is dominated by the Besov like norm $\left(\sum\left\|\Delta_{n} f\right\|_{p}^{2}\right)^{1 / 2}$ in this range.

Consider for a moment the real Walsh polynomials of degree $d$. So, let $\Delta_{n} e=$ $\prod_{i=1}^{d} \varepsilon_{n_{i}}\left(\omega_{i}\right), \omega=\left(\omega_{1}, \ldots, \omega_{d}\right) \in[0,1)^{d}$, with $\varepsilon_{n}$ the $n$th Rademacher function. Then $g=\sum c_{n} \Delta_{n} e$ is a dyadic martingale for any constants $c_{n}$. Borell [2] first obtained (1.1) in case $f$ takes the form $g$. Note that his proof relies on the twopoint contraction-type estimate [1].

$$
\left[\frac{1}{2}\left(|a-b / \sqrt{p-1}|^{p}+|a+b / \sqrt{p-1}|^{p}\right)\right]^{1 / p} \leq \sqrt{a^{2}+b^{2}}, \quad p>2 .
$$

The proof of Theorem 1, below in $\S 2$, relies also on (1.2). However, whereas, due to the independence property of the Rademacher functions, the proof in [2] is valid

Received by the editors July 21, 1986. Presented to Conference on "Dependence in Probability and Statistics", Oberwolfach (1985).

1980 Mathematics Subject Classification (1985 Revision). Primary 60G99.

Key words and phrases. d-parameter dyadic martingale. 
in fact for Banach space valued coefficients $c_{n}$ in the Walsh polynomial $g$, our proof of Theorem 1 utilizes in general only the orthogonality of the differences $\Delta_{n} f$, so does not extend to the Banach space valued case.

We prove first

$$
\|f\|_{p} \leq(p-1)^{d / 2} \sqrt{\sum\left\|\Delta_{n} f\right\|_{p}^{2}}, \quad p \geq 2 .
$$

The case $d \geq 2$ in (1.3) follows immediately from the case $d=1$ by induction since the marginal difference array $\left\{\sum_{n_{d}=1}^{\infty} \Delta_{n} f ; n \in Z_{+}^{d}\right\}$ defines a $(d-1)$ parameter dyadic martingale for each fixed $d$ th coordinate of the argument. Minkowski's inequality is needed in the induction step. It is also instrumental in establishing the initial case. Relation (1.1) follows from (1.3) by Doob's inequality.

Note that (1.3) is sharp in the following sense. Denote by $C_{p, d}$ the best constant in the norm inequality (1.3). Then

$$
\lim _{p \rightarrow \infty}\left(\log C_{p, d}\right) /(d \log p)=\frac{1}{2} .
$$

This can be seen directly by choosing for each positive integer $N$

$$
f=f_{N}=\sum^{+} \Delta_{n} e
$$

where the + sign indicates that the sum is extended over all $n \in[1, N]^{d}$ such that $n_{i}<n_{j}$ when $i<j$. Note that when $d=2, f=\left(\left(\sum_{i=1}^{N} \varepsilon_{i}\right)^{2}-N\right) / 2$. More generally one can show that as $N \rightarrow \infty$,

$$
\|f\|_{p} \sim\left\|\left(\sum_{i=1}^{N} \varepsilon_{i}\right)^{d}-N^{d / 2}\right\|_{p} / d !
$$

where by convergence of moments in the central limit theorem this last expression is asymptotically no less than

$$
\left(-1+E^{1 / p}|\mathcal{N}(0,1)|^{d p}\right) N^{d / 2} / d !
$$

On the other hand, $\sum^{+}\left\|\Delta_{n} e\right\|_{p}^{2} \sim N^{d} / d$ ! as $N \rightarrow \infty$. Hence,

$$
\begin{aligned}
2 \liminf _{p \rightarrow \infty}\left(\log C_{p, d}\right) /(d \log p) & \geq \liminf _{p \rightarrow \infty}\left(-\log (d !)+\frac{2}{p} \log E|\mathcal{N}(0,1)|^{d p}\right) /(d \log p) \\
& =\lim _{p \rightarrow \infty}(-d \log (d)+d \log (d p)) /(d \log p)=1 .
\end{aligned}
$$

2. Proof of Theorem 1. Let first $d=1$. Following [2], write $\Omega=\{-1,1\}^{N}$ and

$$
d \mu_{i}(x)=\frac{\delta_{-1}+\delta_{1}}{2}\left(x_{i}\right), \quad x=\left(x_{1}, \ldots, x_{n}, \ldots\right) \in \Omega .
$$

Put $\mu_{\infty}=\prod_{i \in \mathbf{N}} \mu_{i}$ and note that $\left(\Omega, \mu_{\infty}\right)$ is isomorphic to the Lebesgue unit interval. Let now $\left\{f_{n}\right\}$ be an $L^{2}$ bounded one-parameter dyadic martingale. Thus, $f_{n}=f_{n}\left(x_{1}, \ldots, x_{n}\right)=E^{\mu_{\infty}}\left(f / x_{1}, \ldots, x_{n}\right)$ with some $f \in L^{2}\left(\Omega, \mu_{\infty}\right)$. Introduce also $K(x, \xi)=1+\lambda x \xi$, and define for any function $g$ on $\Omega$ :

$$
K_{m}^{*} g=\int K\left(x_{m}, \xi_{m}\right) g\left(x_{1}, \ldots, x_{m-1}, \xi_{m}, x_{m+1}, \ldots\right) d \mu_{\infty}(\xi) .
$$


Since $\left\{f_{n}\right\}$ is dyadic,

$$
\frac{1}{2}\left[f_{n}\left(x_{1}, \ldots, x_{n-1}, 1\right)+f_{n}\left(x_{1}, \ldots, x_{n-1},-1\right)\right]=f_{n-1}\left(x_{1}, \ldots, x_{n-1}\right) .
$$

It follows that

$$
K_{n}^{*}\left(f_{n}-f_{n-1}\right)=\lambda\left(f_{n}-f_{n-1}\right)=\lambda\left(f_{n}-f_{n-1}\right) .
$$

By (1.2) the integral operator $K_{n}^{*}$ has the important property

$$
\left\|K_{n}^{*} g\right\|_{p, \mu_{n}} \leq\|g\|_{2, \mu_{n}}
$$

with $\lambda=(p-1)^{-1 / 2}$ and $p \geq 2$.

Put now $\Delta_{n} f=f_{n}-f_{n-1}$, fix $p>2$ and set $\lambda=(p-1)^{-1 / 2}$. Notice that if a function $h$ depends only on coordinates $x_{1}, \ldots, x_{m}$ then $K_{n}^{*} h=h$ for $n>m$. Thus,

Hence by (2.2) and orthogonality,

$$
\sum_{m=1}^{n} K_{m}^{*} \Delta_{m} f=K_{n}^{*}\left[\left(\sum_{m=1}^{n} K_{m}^{*} \Delta_{m} f\right)+\Delta_{n} f\right] .
$$

$$
\begin{aligned}
& \left\|\sum_{m=1}^{n} K_{m}^{*} \Delta_{m} f\right\|_{p, \mu_{\infty}}^{2} \\
& \quad \leq\left[\int \cdots \int d \mu_{1} \cdots d \mu_{n-1}\left(\int\left|\sum_{m=1}^{n-1} K_{m}^{*} \Delta_{m} f+\Delta_{n} f\right|^{2} d \mu_{n}\right)^{p / 2}\right]^{2 / p} \\
& \left.\quad \leq\left[\int \cdots \int d \mu_{1} \cdots d \mu_{n-1} \int\left(\left|\sum_{m=1}^{n-1} K_{m}^{*} \Delta_{m} f\right|^{2}+\left|\Delta_{n} f\right|^{2}\right) d \mu_{n}\right]^{p / 2}\right]^{2 / p} .
\end{aligned}
$$

Next by an application of Minkowski's integral inequality, this last expression does not exceed

$$
\begin{gathered}
{\left[\int d \mu_{n}\left(\int \cdots \int\left|\sum_{m=1}^{n-1} K_{m}^{*} \Delta_{m} f\right|^{p} d \mu_{1} \cdots d \mu_{n-1}\right)\right]^{2 / p}} \\
+\left[\int d \mu_{n}\left(\int\left|\Delta_{n} f\right|^{p} d \mu_{1} \cdots d \mu_{n-1}\right)\right]^{2 / p}
\end{gathered}
$$

Now repeat this step. The first term of the above expression does not exceed

$$
\begin{gathered}
{\left[\int d \mu_{n-1}\left(\int\left|\sum_{m=1}^{n-2} K_{m}^{*} \Delta_{m} f\right|^{p} d \mu_{1} \cdots d \mu_{n-2}\right)\right]^{2 / p}} \\
+\left[\int d \mu_{n-1}\left(\int\left|\Delta_{n-1} f\right|^{p} d \mu_{1} \cdots d \mu_{n-2}\right)\right]^{2 / p} .
\end{gathered}
$$

Continuing in this way, obtain that

$$
\begin{aligned}
\left\|\sum_{m=1}^{n} K_{m}^{*} \Delta_{m} f\right\|_{p, \mu_{\infty}}^{2} & \leq \sum_{m=1}^{n}\left[\int d \mu_{m}\left(\int \cdots \int\left|\Delta_{m} f\right|^{p} d \mu_{1} \cdots d \mu_{m-1}\right)\right]^{2 / p} \\
& =\sum_{m=1}^{n}\left\|\Delta_{m} f\right\|_{p}^{2} .
\end{aligned}
$$


By (2.1) and (2.3), (1.3) holds in case $d=1$. By the remarks of the introduction we conclude that (1.3) holds in the general case. Here $\|f\|_{p}=\sup _{\nu}\left\|f_{\nu}\right\|_{p}$.

To obtain (1.1) note that in case $d=2$,

$$
\max _{\nu}\left|f_{\nu}\right|=\sup _{m}\left(\sup _{n}\left|f_{m n}\right|\right),
$$

and that $g_{m}=\sup _{n}\left|f_{m n}\right|$ forms a submartingale in $m$ relative to the fields $\mathcal{F}_{m \infty}$. Indeed, $g_{m}$ is an increasing limit of positive submartingales: $g_{m}=\lim _{n \rightarrow \infty}\left|f_{m 1}\right| \mathrm{V}$ $\cdots \vee\left|f_{m n}\right|$. This observation goes back to Cairoli [3]. Hence, by Doob's inequality,

$$
\begin{aligned}
\left\|f^{*}\right\|_{p} & =\left\|\sup _{m} g_{m}\right\|_{p} \leq q \sup _{m}\left\|g_{m}\right\|_{p} \\
& =q \sup _{m}\left\|\sup _{n}\left|f_{m, n}\right|\right\|_{p} \\
& \leq q^{2} \sup _{m} \sup _{n}\left\|f_{m, n}\right\|_{p}=q^{2}\|f\|_{p} .
\end{aligned}
$$

Thus, by (1.3) the proof of Theorem 1 is complete.

\section{REFERENCES}

1. W. Beckner, Inequalities in Fourier analysis, Ann. of Math. 102 (1975), 159-182.

2. C. Borell, On the integrability of Banach space valued Walsh polynomials, Lecture Notes in Math., vol. 721, Springer-Verlag, 1979, pp. 1-3.

3. R. Cairoli, Une inégalité pour martingales à indices multiples et ses applications, Lecture Notes in Math., vol. 124, Springer-Verlag, 1970, pp. 1-27.

4. R. Gundy, Notes, 1980.

Department of Mathematics, Washington University, St. Louis, Missouri 63130

Current address: Department of Mathematics, University of Colorado at Colorado Springs, Colorado Springs, Colorado 80933 